\title{
Moment of inertia of a trapped superfluid gas of atomic fermions
}

\author{
M. Farine, ${ }^{1}$ P. Schuck, ${ }^{2}$ and X. Viñas ${ }^{3}$ \\ ${ }^{1}$ Ecole Navale, Lanvéoc-Poulmic, 29240 Brest-Naval, France \\ ${ }^{2}$ Institut des Sciences Nucléaires, Université Joseph Fourier, CNRS-IN2P3, 53, Avenue des Martyrs, \\ F-38026 Grenoble Cedex, France \\ ${ }^{3}$ Departament d'Estructura i Constituents de la Matéria Facultat de Física, Universitat de Barcelona Diagonal 647 , \\ E-08028 Barcelona, Spain
}

(Received 22 December 1998; revised manuscript received 14 June 1999; published 16 June 2000)

\begin{abstract}
The moment of inertia $\Theta$ of a trapped superfluid gas of atomic fermions $\left({ }^{6} \mathrm{Li}\right)$ is calculated as a function of the temperature. At zero temperature the moment of inertia takes on the irrotational-flow value. Only for $T$ very close to $T_{c}$ is rigid rotation attained. It is proposed that future measurements of the rotational energy will unambiguously reveal whether the system is in a superfluid state or not.

PACS number(s): 03.75.Fi, 74.20.Fg
\end{abstract}

\section{INTRODUCTION}

The advent in 1995 of Bose-Einstein condensation of atomic bosons in magnetic traps certainly represents a milestone in the study of bosonic many-body quantum systems. This is so because a systematic study of these systems, starting with the free-particle case, as a function of increasing density, particle number, and other system parameters, seems possible and has already progressed to a large extent while going on at a rapid pace $[1,2]$. The recent experimental achievement of trapping ${ }^{6} \mathrm{Li}$ atoms and other fermionic alkali-metal atoms [3] gives hope that as much progress will be made in the near future for the fermionic many-body problem as for the bosonic systems. Indeed, the first FermiDirac degeneracy of trapped ${ }^{40} \mathrm{~K}$ atoms has already been observed (see De Marco and Jin [4]). In this reference more of the physics of trapped fermionic atoms is also discussed. For atoms with attractive interaction one can envisage that the trapped system undergoes a transition to the superfluid state. For instance, ${ }^{6} \mathrm{Li}$ atoms can be trapped in two different hyperfine states. In the spin-polarized case the $s$-wave interaction turns out to be very strong and attractive (scattering length $a=-2063 a_{0}$ with $a_{0}$ the Bohr radius) favoring a phase transition to the superfluid state. This possibility has recently provoked a number of theoretical investigations (see [5] for a more detailed discussion of a possible superfluid state). One major question that is under debate is how to detect the superfluidity of such a fermionic system, since in contrast to a bosonic system the density of a fermionic system is scarcely affected by the transition to the superfluid state [6]. Several proposals such as the study of the decay rate of the gas or of the scattering of atoms off the gas have been advanced [5]. Although such investigations may give valuable indications of a possible superfluid phase, we think that, in analogy with nuclear physics, a measurement of the moment of inertia certainly would establish an unambiguous signature of superfluidity. To measure the spin and rotational energy of trapped atoms definitely is a great challenge for the future. However, in nuclear physics, where $\gamma$ spectroscopy is extremely well developed, the strong reduction of the moment of inertia with respect to its rigid-body value has been considered as a firm indicator of nucleon superfluidity since immediately after the discovery of nuclear rotational states almost half a century back [6]. Therefore, while awaiting future experimental achievements for trapped fermionic atoms also, it is our intention in this work to give some theoretical estimates of the moment of inertia as a function of deformation of the traps or temperature of the gas. In this study we can greatly profit from the experience nuclear physicists have accumulated over recent decades in describing such phenomena. The expectation is indeed that there will be a great analogy between the physics of confined atomic fermions and what one calls in nuclear physics the liquid-drop part of the nucleus. As astonishing as it may seem, assemblies of fermions containing no more than $\sim 200$ particles (nucleons) already exhibit an underlying macroscopic structure well known from the Bethe-Weizsäcker formula for nuclear masses [6]. In superfluid rotating nuclei Migdal proposed as early as 1959 a statistical description of the nuclear moment of inertia [7] that grasped the essential physics of a self-contained rotating superfluid fermi liquid drop and which serves as a reference even today.

In the present work we will cast Migdal's approach into the more systematic language of the Thomas-Fermi theory which together with its extensions has long been applied to normal-fluid but also to superfluid nuclei $[6,8,9]$. It is fortunate that we can profit from this experience for the description of trapped fermions, since their number, of order $10^{5}$, together with the smoothness of the potential, certainly turns a statistical description into a very precise tool. On the other hand, it cannot be excluded that in the future much smaller systems of trapped atomic fermions with numbers $\sim 10^{2}$ may be studied, probably revealing many analogies with nuclei, such as shell structure, etc. The investigation of the transition from microscopic to macroscopic as the number of particles is increased continuously may then become a very interesting field in the case of atomic fermions also. In detail, our paper is organized as follows. In Secs. II and III we review the Thomas-Fermi approach to inhomogeneous superfluid Fermi systems. In Sec. IV first the so-called Inglis part of the moment of inertia of a rotating superfluid and confined gas of atomic fermions is presented. Second, the influence of the reaction of the pair field on the moment of inertia is calculated. It is shown that this leads to the irrotational-flow value 
in the limit of strong pairing. In Sec. V the current distributions in the superfluid and normal-fluid regimes are contrasted. In Sec. VI the numerical results are presented in detail together with discussion and conclusions.

\section{THOMAS-FERMI APPROACH TO FERMIONIC ATOMS IN DEFORMED TRAPS}

The Thomas-Fermi (TF) approach to trapped gases of atomic bosons is a well-accepted practice [2]. For trapped atomic fermions the same approximation applies in different conditions. It has, however, recently also been applied to this kind of situation [5]. The TF approach for fermions is also extensively applied to other finite systems such as atomic nuclei, metallic clusters, etc. The smallness parameter is given by

$$
\eta=\frac{\nabla V(r)}{k_{F}(r) V(r)},
$$

where $V$ is the mean-field potential and $k_{F}(r)$ the local Fermi momentum,

$$
k_{F}(r)=\sqrt{\left(2 m / \hbar^{2}\right)\left[\varepsilon_{F}-V(r)\right]} .
$$

With a typical frequency of the external harmonic potential of $\omega_{0}=7 \mathrm{nK}$ and $\varepsilon_{F}=600 \mathrm{nK}$, one realizes that $\eta \ll 1$ up close to the end of the classically allowed region. For integrated quantities the region around the classical turning point carries little weight and therefore the TF approximation for a number of trapped atoms of the order of $10^{5}$ is certainly very well justified.

Furthermore, as in the boson case, the TF approach [6] to trapped atomic gases becomes extremely simple because the large interparticle distance makes a pseudopotential approximation to atomic interactions valid. Let us therefore write down the TF equation for a doubly-spin-polarized system of trapped $\left({ }^{6} \mathrm{Li}\right)$ atoms in the normal-fluid state. For convenience we first consider the system at zero temperature $T$, discussing the $T \neq 0$ case later on. In the TF approximation the distribution function for particles in each spin state is given by

$$
f(\mathbf{R}, \mathbf{p})=\theta\left(\mu-H_{\mathrm{cl}}\right)
$$

with

$$
H_{\mathrm{cl}}=\frac{p^{2}}{2 m}+V_{\mathrm{ex}}(\mathbf{R})-g \rho(\mathbf{R})
$$

(in this work we consider only equal occupation of both spin states). Here $\mu$ is the chemical potential, and $V_{\mathrm{ex}}(\mathbf{R})$ stands for the trap potential, which is supposed to be of harmonic form. The density $\rho(\mathbf{R})$ is obtained from the self-consistency equation,

$$
\rho(\mathbf{R})=\int \frac{d^{3} p}{(2 \pi \hbar)^{3}} f(\mathbf{R}, \mathbf{p})=\frac{1}{6 \pi^{2}} k_{F}^{3}(\mathbf{R}),
$$

with

$$
k_{F}(\mathbf{R})=\sqrt{\left(2 m / \hbar^{2}\right)\left[\mu-V_{\mathrm{ex}}(\mathbf{R})+g \rho(\mathbf{R})\right]}
$$

the local Fermi momentum. The coupling constant $g$ is related to the scattering length in the same way as in the case of Bose condensed gases $[1,2]$ via

$$
g=\frac{4 \pi \hbar^{2}|a|}{m} .
$$

The TF equation (2.5) leads to a cubic equation for the selfconsistent density, which can be solved straightforwardly as a function of the external potential. In this paper our main interest will be the study of the moment of inertia of a rotating condensate. Since the study is very much simplified by assuming that the self-consistent potential is again a harmonic oscillator and since the effect of the attractive interaction between the atoms essentially results in a narrowing of the self-consistent potential with respect to the external one, we will use instead of the exact TF solution for the density the following trial ansatz for the local Fermi momentum:

$$
k_{F}^{\text {trial }}(\mathbf{R})=\sqrt{\left(2 m / \hbar^{2}\right)\left[\mu-(m / 2)\left(\omega_{x}^{2} R_{x}^{2}+\omega_{y}^{2} R_{y}^{2}+\omega_{z}^{2} R_{z}^{2}\right)\right]},
$$

where $\omega_{x}, \omega_{y}$, and $\omega_{z}$ are the variational parameters. The chemical potential is determined from the particle number condition

$$
N=\int d^{3} r \rho^{\text {trial }}(r)
$$

and the kinetic energy density is given by

$$
\tau(\mathbf{R})=\int \frac{d^{3} p}{(2 \pi \hbar)^{3}} \frac{p^{2}}{2 m} f(\mathbf{R}, \mathbf{p})=\frac{1}{10 \pi^{2}}\left[k_{F}^{\text {trial }}(\mathbf{R})\right]^{5} .
$$

We can then analytically calculate the total energy

$$
E\left(\omega_{x}, \omega_{y}, \omega_{z}\right)=\int d^{3} R\left(\tau(\mathbf{R})+V_{\mathrm{ex}}(\mathbf{R}) \rho(\mathbf{R})-\frac{g}{2} \rho^{2}(\mathbf{R})\right)
$$

as a function of $\omega_{x}, \omega_{y}$, and $\omega_{z}$. Minimizing this expression with respect to $\omega_{x}, \omega_{y}$, and $\omega_{z}$ for a given external deformed harmonic oscillator potential

$$
V_{\mathrm{ex}}=\frac{m}{2}\left(\omega_{0 x}^{2} R_{x}^{2}+\omega_{0 y}^{2} R_{y}^{2}+\omega_{0 z}^{2} R_{z}^{2}\right)
$$

leads to the variational solution. For the spherical case $\omega_{x}$ $=\omega_{y}=\omega_{z}=\omega$, this is shown in Fig. 1. We see that this approximation to the TF equation is quite reasonable. For an external harmonic potential with frequency $\nu=\omega_{0} / 2 \pi$ $=144 \mathrm{~Hz}$ or $\hbar \omega_{0} / k_{B}=6.9 \mathrm{nK}$, corresponding to the conditions of the experiment of Bradley and co-workers [10], the variational frequency is $\hbar \omega / k_{B}=7.69 \mathrm{nK}$. Since $\omega>\omega_{0}$ this implies a compression of the density. Increasing $\omega$ by $6 \%$ $\left(\hbar \omega / k_{B}=8.21 \mathrm{nK}\right)$ from its variational value allows an almost perfect reproduction of the full TF solution. We will 


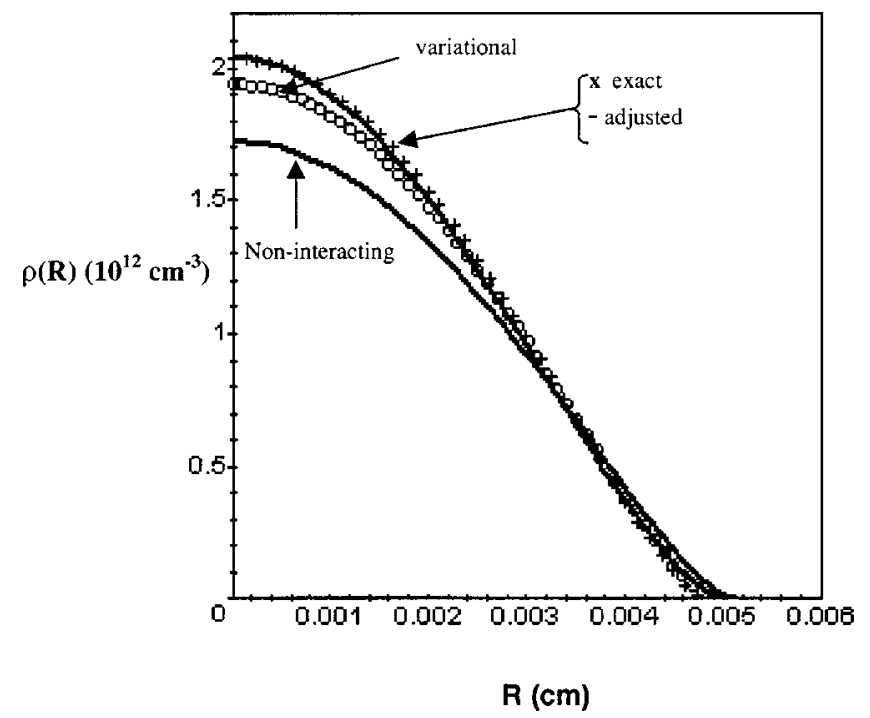

FIG. 1. Density profiles, for the case of a spherical trap, of the noninteracting case (full line) and the interacting case calculated once exactly from Eq. (2.5) (crosses) with $V_{\text {ex }}$ given by Eq. (2.12), and once using the variationally determined harmonic-oscillator potential (open squares). Squeezing the variational $\omega$ by $6 \%$ yields a density that lies on top of the exact TF solution.

adopt this latter value in all our forthcoming calculations. The experimental situation for the rotating deformed case is such that the rotation of the trap is performed around the $x$ axis (the long axis), permitting slight triaxial deformations in the plane perpendicular to the $x$ axis that is in the $y-z$ plane. In order to simulate such an experimental situation we simply first make a volume-conserving

$$
\left(\omega_{\perp}\right)^{2} \omega_{x}=\omega^{3}
$$

prolate deformation around the $x$ axis,

$$
\begin{gathered}
\sigma=\frac{\omega_{x}}{\omega_{\perp}}, \\
\omega_{\perp}=\omega \sigma^{-1 / 3}, \quad \omega_{\perp}=\omega_{y}=\omega_{z}, \\
\omega_{x}=\omega \sigma^{2 / 3} .
\end{gathered}
$$

In order to increase the central density there is interest in making rather strong eccentricities and $\omega_{x} / \omega_{\perp}=\frac{1}{8}$ is a typical value which we will adopt in this paper. In a second step we fix $\omega_{x}$ and deform around the $x$ axis, again keeping the volume fixed. We define the deformation parameter as

$$
\delta=\frac{\omega_{z}}{\omega_{y}}
$$

We finally have the two-parameter deformation

$$
\begin{gathered}
\omega_{x}=\omega \sigma^{2 / 3}, \\
\omega_{y}=\omega \sigma^{-1 / 3} \delta^{-1 / 2}, \\
\omega_{z}=\omega \sigma^{-1 / 3} \delta^{1 / 2},
\end{gathered}
$$

with $\sigma \ll \delta<1$. From now on we will therefore use for the nonsuperfluid Wigner function at zero temperature the expression

$$
f(\mathbf{R}, \mathbf{p})=\theta\left(\mu-\frac{p^{2}}{2 m}-V(\mathbf{R})\right),
$$

with

$$
V(\mathbf{R})=\frac{m}{2}\left(\omega_{x}^{2} R_{x}^{2}+\omega_{y}^{2} R_{y}^{2}+\omega_{z}^{2} R_{z}^{2}\right)
$$

$\omega_{x}, \omega_{y}, \omega_{z}$ from Eq. (2.17), and $\mu$ determined from the particle number condition.

\section{SUPERFLUID CASE}

Since, as described in the Introduction, trapped spinpolarized ${ }^{6} \mathrm{Li}$ atoms, in different hyperfine states, experience a strong attractive $s$-wave interaction, the system very likely will undergo a transition to the superfluid state at some critical temperature $T_{c}$ as was discussed in detail in Ref. [5]. As we have pointed out in the Introduction, the superfluid state will unambiguously reveal itself in the value of its moment of inertia. At present measurement of the angular momenta of trapped Bose or Fermi gases has not been achieved and represents a future challenge to the experimenters. In order to establish how the two essential system parameters, which are the value of the gap (that is, in fact, the temperature which triggers the gap) and the deformation of the external trap influence the value of the moment of inertia, we will now proceed to its evaluation in the superfluid state.

Since we are dealing with an inhomogeneous system, even in the nonrotating case the gap is actually a nonlocal quantity $\Delta\left(\mathbf{r}, \mathbf{r}^{\prime}\right)$ or in Wigner space $\Delta(\mathbf{R}, \mathbf{p})$. We will find later that at zero temperature the coherence length of the Cooper pair, $\xi=\hbar^{2} k_{F} / m \Delta$, is larger than the oscillator length $l=\sqrt{\hbar / m \omega_{0}} \approx\left(0.63 \times 10^{5}\right) a_{0}$ with $a_{0}$ the Bohr radius. We therefore have to be careful in applying the TF theory for temperatures $T$ much lower than the critical temperature $T_{c}$ where the gap vanishes. We will discuss this point more thoroughly later and in the Appendix. We therefore go on and apply the TF approximation to the superfluid state. It has been shown in $[9,6]$ that to lowest order in $\hbar$ the gap equation is given by

$$
\Delta(\mathbf{R}, \mathbf{p})=\int \frac{d^{3} k}{(2 \pi \hbar)^{3}} v(\mathbf{p}, \mathbf{k}) \frac{\Delta(\mathbf{R}, \mathbf{k})}{2 E(\mathbf{R}, \mathbf{k})} \tanh \left(\frac{E(\mathbf{R}, \mathbf{k})}{2 T}\right),
$$

where $E(\mathbf{R}, \mathbf{p})$ is the quasiparticle energy,

$$
E(\mathbf{R}, \mathbf{p})=\left[\left(\frac{p^{2}-p_{F}^{2}(\mathbf{R})}{2 m^{*}(\mathbf{R})}\right)^{2}+\Delta^{2}(\mathbf{R}, \mathbf{p})\right]^{1 / 2}
$$

with $p_{F}(\mathbf{R})=\hbar k_{F}(\mathbf{R})$ the local Fermi momentum (2.6). Since the effective mass $m^{*}$ is so far unknown for trapped gases of atomic fermions we will take $m^{*}=m$. Furthermore, for the time being, as in [5], we will eliminate the inter- 


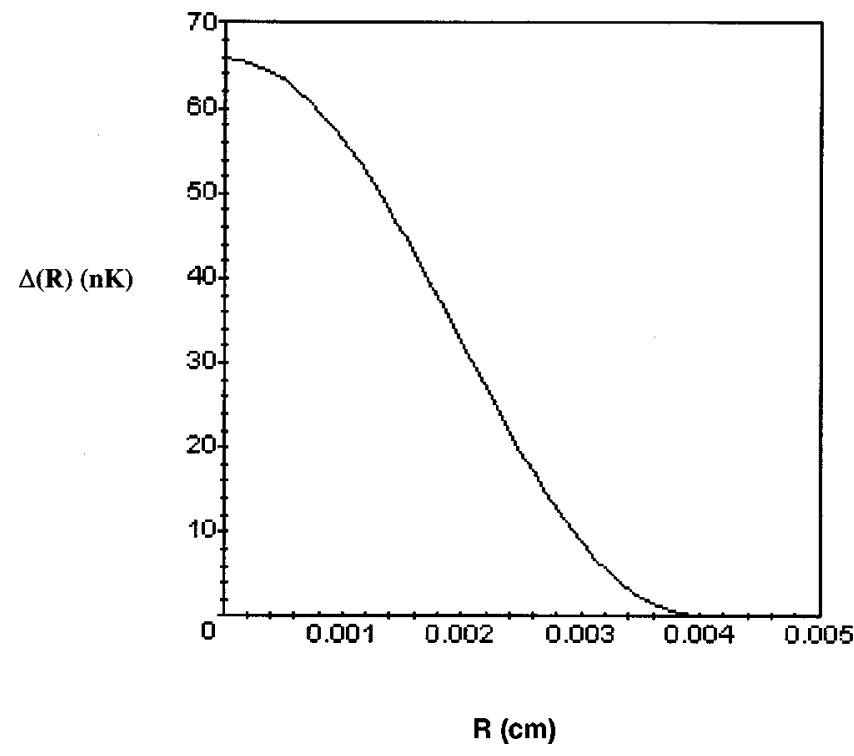

FIG. 2. The gap for a spherical trap as a function of the radius.

atomic potential $v$ in Eq. (3.1), expressing it by the scattering length (2.7). We then obtain [5]

$$
\begin{aligned}
\Delta(\mathbf{R}, \mathbf{p})= & g \int \frac{d^{3} k}{(2 \pi \hbar)^{3}}\left(\frac{\tanh [E(\mathbf{R}, \mathbf{k}) / 2 T]}{2 E(\mathbf{R}, \mathbf{k})}\right. \\
& \left.-\frac{P}{2\left[\varepsilon_{k}-\varepsilon_{F}(\mathbf{R})\right]}\right) \Delta(\mathbf{R}, \mathbf{k}),
\end{aligned}
$$

where $P$ stands for principal value, $\varepsilon_{k}=\hbar^{2} k^{2} / 2 m$, and $\varepsilon_{F}$ $=\hbar^{2} k_{F}^{2} / 2 m$. At zero temperature, as described in [11], Eq. (3.3) can be solved analytically in the limit $\Delta\left(\mathbf{R}, p_{F}(\mathbf{R})\right) / \varepsilon_{F}(\mathbf{R}) \rightarrow 0$. The result is given by

$$
\Delta_{F}(\mathbf{R}) \equiv \Delta\left(\mathbf{R}, k_{F}(\mathbf{R})\right)=8 e^{-2} \varepsilon_{F}(\mathbf{R}) e^{-\left[\pi / 2 k_{F}(\mathbf{R})|a|\right]} .
$$

A posteriori one can verify that $\Delta_{F} / \varepsilon_{F} \ll 1$ for all values of $R$ and therefore Eq. (3.4) is an excellent approximation to Eq. (3.3). This has also been found in [5]. For $286500{ }^{6} \mathrm{Li}$ atoms, the case considered in [5], the gap is shown for a spherical trap as a function of the radius in Fig. 2.

For determination of the critical temperature $T_{c}$ and, later on, for the moment of inertia we will need the value $\Delta$ of the gap at the Fermi energy. Since the detailed level structure at the Fermi energy is unknown and in fact unimportant, we will consider the gap $\Delta\left(\varepsilon_{F}\right)$ averaged over the states at the Fermi energy,

$$
\Delta\left(\varepsilon_{F}\right) \equiv \Delta=\operatorname{Tr}\left[\hat{\Delta} \hat{\rho}\left(\varepsilon_{F}\right)\right]
$$

with

$$
\hat{\rho}(\varepsilon)=\frac{1}{g(\varepsilon)} \sum_{n}|n\rangle\langle n| \delta\left(\varepsilon-\varepsilon_{n}\right)=\frac{1}{g(\varepsilon)} \delta(\varepsilon-H),
$$

where $|n\rangle$ and $\varepsilon_{n}$ are the states and energies of the harmonic oscillator with frequency $\omega$ and

$$
g(\varepsilon)=\sum_{n} \delta\left(\varepsilon-\varepsilon_{n}\right)=\operatorname{Tr} \delta(\varepsilon-H)
$$

is the level density.

It has been shown in [12] that again the TF approximation leads to an excellent average value,

$$
\Delta=\frac{1}{g^{\mathrm{TF}}\left(\varepsilon_{F}\right)} \int \frac{d^{3} R d^{3} p}{(2 \pi \hbar)^{3}} \Delta_{F}(\mathbf{R}) \delta\left(\varepsilon_{F}-H_{\mathrm{cl}}\right)
$$

In the spherical case with $\Delta_{F}(\mathbf{R})$ from Eq. (3.4) all integrals but the radial one can be performed analytically, the last being done numerically. For the case shown in Fig. 2 one obtains

$$
\Delta=16.4 \mathrm{nK}
$$

Quantum-mechanically the BCS equations should be solved in the self-consistent Hartree-Fock (HF) basis and then $T_{c}$ is a global parameter which must be determined from the solution of the quantum-mechanical gap equation. Since we believe that the value in Eq. (3.9) comes rather close to the quantum-mechanical value of the gap at the Fermi energy, we can obtain $T_{c}$ from the usual BCS weak-coupling relation $[13] \Delta=1.76 T_{c}$ to be

$$
T_{c} \approx 10 \mathrm{nK} \text {. }
$$

From Eq. (3.9) we obtain the coherence length $\xi$ $=\hbar^{2} k_{F} / m \Delta=2 \varepsilon_{F} / \Delta k_{F}$. With $\varepsilon_{F}=983.67 \mathrm{nK}$, which corresponds to our approximate "self-consistent" harmonic solution with $\omega=8.21 \mathrm{nK}$ and $k_{F}|a|=0.56$, one obtains $\xi$ $\approx\left(4 \times 10^{5}\right) a_{0}$ which is about a factor of 7 larger than the oscillator length of the trap (see above) that contains 286500 particles. This seems to invalidate the TF approximation. However, we know by experience that often the TF approximation remains quite reasonable beyond its limit [6]. For example, the conditions of validity in $[9,12]$ for superfluid nuclei are much worse than here and still the results are accurate beyond expectation. We therefore think that the values of Eqs. (3.9) and (3.10) are reasonable estimates for the gap and the critical temperature. In order to check this assumption we give in the Appendix a more refined semiclassical solution of the gap equation, which demands only that the TF approximation in the normal-fluid state is well justified. We find values for $\Delta$ and $T_{c}$ that are $\sim 30 \%$ lower than in Eqs. (3.9) and (3.10). In view of the crudeness of the TF approach, this indicates a quite satisfying consistency between the results.

We also will have to know the detailed $T$ dependence of the gap, $\Delta(T)$, which, however, in BCS theory, given $\Delta(0)$ and $T_{c}$, is determined by the universal function $\Delta(T) / \Delta(0)$ in terms of $T / T_{c}$. This function is determined from the solution of the equation [13]

$$
-\ln \left(\frac{\Delta(T)}{\Delta(0)}\right)=A\left(\frac{\Delta(T)}{T}\right),
$$

with 


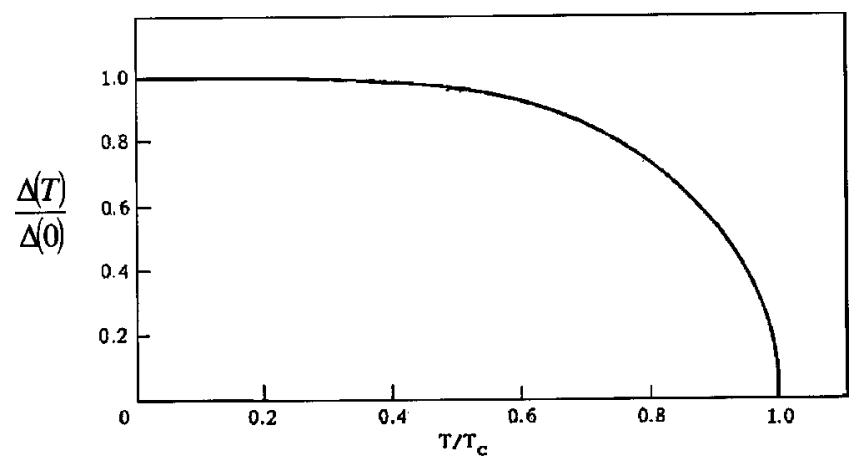

FIG. 3. Ratio of the energy gap to the gap at $T=0 \mathrm{~K}$ as a function of temperature.

$$
A(u)=\int_{0}^{\infty} d y \frac{1}{\sqrt{y^{2}+u^{2}}}\left[1-\tanh \left(\frac{\sqrt{y^{2}+u^{2}}}{2}\right)\right] .
$$

For completeness it is shown in Fig. 3. We will later use this $T$ dependence of the gap for the evaluation of the moment of inertia.

\section{MOMENT OF INERTIA}

The moment of inertia of a rotating nucleus has been fully formulated in linear response theory (i.e. the random-phase approximation) by Thouless and Valatin [14]. The corresponding expression is therefore called, in the nuclear physics literature [6], the Thouless-Valatin moment of inertia. It consists of two parts, the so-called Inglis term, which describes the free-gas response, and the part that accounts for the reaction of the mean field and pair potential to the rotation. In the superfluid case the Inglis part has been generalized by Belyaev [15] and the linear response of the gap parameter to the value of the moment of inertia was first evaluated, together with the Inglis term, by Migdal [7]. The reaction of the HF field to the rotation is a minor effect and we will neglect it in this work. We therefore will write the moment of inertia as a sum of the Inglis-Belyaev term $\Theta_{\mathrm{IB}}$ and the Migdal term $\Theta_{M}$. In total

$$
\Theta=\Theta_{\mathrm{IB}}+\Theta_{M} .
$$

In order to derive an expression for $\Theta$ in linear response theory we will use the Gorkov approach described in detail in many textbooks (in what follows we will use the notation of [16]). Since in addition the derivation of the linear response for $\Theta$ is given in the original article of Migdal [7] and represented in a more elaborate version in [8], we will be very brief here and only give more details where in our opinion the presentations in $[7,8]$ may not be entirely explicit. Let us start by writing down the Gorkov equations in matrix notation,

$$
\begin{gathered}
\left(-\frac{\partial}{\partial \tau}-H+\mu\right) G=1-\Delta F^{+}, \\
\left(\frac{\partial}{\partial \tau}+H^{*}+\mu\right) F^{*}=\Delta^{*} G,
\end{gathered}
$$

with

$$
H=H_{0}-\Omega L_{x} \equiv H_{0}+H_{1},
$$

where now $H_{0}$ is the shell-model Hamiltonian (2.4), or rather the approximate one used in Eqs. (2.18) and (2.19), and

$$
L_{x}=r_{y} p_{z}-r_{z} p_{y}
$$

the angular momentum operator corresponding to a rotation with angular frequency $\Omega$ around the $x$ axis. In Eq. (4.2) $G$ and $F$ are the normal and anomalous Matsubara Green's functions (see Chap. 51 of [16])

$$
\begin{gathered}
G_{n n^{\prime}}=-\left\langle T_{\tau} a_{n}(\tau) a_{n^{\prime}}{ }^{+}\left(\tau^{\prime}\right)\right\rangle, \\
F_{n n^{\prime}}^{+}=-\left\langle T_{\tau} a_{n}{ }^{+}(\tau) a_{n^{\prime}}{ }^{+}\left(\tau^{\prime}\right)\right\rangle .
\end{gathered}
$$

Linearizing Eq. (4.2) with respect to $H_{1}$, that is, $G=G_{0}$ $+G_{1}, F^{+}=F_{0}^{+}+F_{1}^{+}$, and $\Delta=\Delta_{0}+\Delta_{1}$ (as mentioned we will neglect the influence of the rotational field on $H_{0}$ ), one obtains for Eq. (4.2)

$$
G_{1}=G_{1 \mathrm{IB}}+G_{1 M},
$$

with

$$
\begin{gathered}
G_{1 \mathrm{IB}}=G_{0} H_{1} G_{0}+F_{0}^{+} H_{1}^{*} F_{0}^{+}, \\
G_{1 M}=-G_{0} \Delta_{1} F_{0}^{+}-F_{0}^{+} \Delta_{1}^{*} G_{0},
\end{gathered}
$$

and

$$
F_{1}^{+}=D_{0} H_{1}^{*} F_{0}^{+}+F_{0}^{+} H_{1} G_{0}-F_{0}^{+} \Delta_{1} F_{0}^{+}+D_{0} \Delta_{1}^{*} G_{0},
$$

where

$$
\begin{gathered}
D=\frac{i \omega_{n}-H_{0}}{\omega_{n}^{2}+H_{0}^{2}+\Delta_{0}^{2}}, \quad G_{0}=\frac{i \omega_{n}+H_{0}}{\omega_{n}^{2}+H_{0}^{2}+\Delta_{0}^{2}}, \\
F_{0}^{+}=\frac{\Delta_{0}}{\omega_{n}^{2}+H_{0}^{2}+\Delta_{0}^{2}},
\end{gathered}
$$

and $\omega_{n}$ are the Matsubara frequencies [16].

In Eqs. (4.5) and (4.7) we have split the first-order Green's function in an obvious notation into the InglisBelyaev and Migdal contributions. For the latter one needs the linear reaction of the pair field to the rotation. We will see later how this can be determined from Eq. (4.7). First, however, let us evaluate the IB part of the moment of inertia.

\section{A. The Inglis-Belyaev part of the moment of inertia}

The IB part of the moment of inertia can be evaluated without knowledge of $\Delta_{1}$, i.e., without the use of Eq. (4.7). The density response corresponding to $G_{1 \mathrm{IB}}$ of Eq. (4.5) is evaluated from the limit $\tau^{\prime} \rightarrow \tau^{+}$or from summing over the Matsubara frequencies in the upper half plane (see Chap. 7 of [16]). One obtains the well-known result $[6-8,15,16]$

$$
\left(\rho_{1 \mathrm{IB}}\right)_{n n^{\prime}}=\left\langle n\left|L_{x}\right| n^{\prime}\right\rangle F_{n n^{\prime}},
$$


with

$$
F=F_{+}\left(1-f-f^{\prime}\right)+F_{-}\left(f-f^{\prime}\right)
$$

where

$$
\begin{gathered}
F_{ \pm}=F_{ \pm}\left(\varepsilon_{n}, \varepsilon_{n^{\prime}}\right)=\frac{E_{n} E_{n^{\prime}} \mp \xi_{n} \xi_{n^{\prime}}-\Delta\left(\varepsilon_{n}\right) \Delta\left(\varepsilon_{n^{\prime}}\right)}{2 E_{n} E_{n^{\prime}}\left(E_{n} \pm E_{n^{\prime}}\right)}, \\
f=f\left(\varepsilon_{n}\right)=\frac{1}{1+e^{E_{n} / T}}, \quad f^{\prime}=f\left(\varepsilon_{n}^{\prime}\right),
\end{gathered}
$$

and

$$
E_{n}=\sqrt{\xi_{n}^{2}+\Delta^{2}\left(\varepsilon_{n}\right)}, \quad \xi_{n}=\varepsilon_{n}-\mu
$$

are the quasiparticle energies with $\varepsilon_{n}$ the energies of the harmonic-oscillator potential (2.19). The gap parameters $\Delta_{n}$ have been replaced in Eq. (4.10), in analogy to Eq. (3.5), to statistical accuracy by $\Delta\left(\varepsilon_{n}\right)$, the ones averaged over the energy shell. The moment of inertia is given by

$$
\Theta_{\mathrm{IB}}=\operatorname{Tr}\left(L_{x} \rho_{1 \mathrm{IB}}\right)
$$

Since we are interested in temperatures $T \leqslant T_{c}$, which are very low with respect to the Fermi energy, we checked that one can to very good accuracy neglect in Eq. (4.9) the thermal factors (4.11). The only important temperature dependence of the moment of inertia therefore exists via the $T$ dependence of the gap. We thus will henceforth treat all formulas as in the $T=0$ limit keeping, however, the $T$ dependence of the gap. With this in mind we can write for the moment of inertia

$$
\begin{aligned}
\Theta_{\mathrm{IB}}= & \sum_{n n^{\prime}} \iint d \omega d \omega^{\prime} \delta\left(\omega-\varepsilon_{n}\right) \\
& \times \delta\left(\omega^{\prime}-\varepsilon_{n^{\prime}}\right)\left|\left\langle n\left|L_{x}\right| n^{\prime}\right\rangle\right|^{2} F_{+}\left(\omega, \omega^{\prime}\right) .
\end{aligned}
$$

In this formula the important quantity to calculate to statistical accuracy is

$$
\begin{aligned}
L_{x}^{2}\left(n, n^{\prime}\right) & \equiv\left|\left\langle n\left|L_{x}\right| n^{\prime}\right\rangle\right|^{2}=\operatorname{Tr}\left[\left(L_{x}\right)\left(\left|n^{\prime}\right\rangle\left\langle n^{\prime}\left|L_{x}\right| n\right\rangle\langle n|\right)\right] \\
& =\int \frac{d^{3} R d^{3} p}{(2 \pi \hbar)^{3}}\left(L_{x}\right)_{W}\left[\left|n^{\prime}\right\rangle\left\langle n^{\prime}\left|L_{x}\right| n\right\rangle\langle n|\right]_{W},
\end{aligned}
$$

where $O_{W} \equiv O(\mathbf{R}, \mathbf{p})$ means the Wigner transform of the operator $O[6]$. To this purpose we again replace the density matrices $|n\rangle\langle n|$ and $\left|n^{\prime}\right\rangle\left\langle n^{\prime}\right|$ by their average on the energy shell (3.6),

$$
|n\rangle\langle n| \rightarrow \hat{\rho}\left(\varepsilon_{n}\right) .
$$

We therefore obtain

$$
\begin{aligned}
\Theta_{\mathrm{IB}}= & \iint d \omega d \omega^{\prime} \\
& \times \int \frac{d^{3} R d^{3} p}{(2 \pi \hbar)^{3}}\left\{\left(L_{x}\right)_{W}\left[L_{x}\left(\omega, \omega^{\prime}\right)\right]_{W}\right\} F_{+}\left(\omega, \omega^{\prime}\right),
\end{aligned}
$$

with

$$
\left[L_{x}\left(\omega, \omega^{\prime}\right)\right]_{W}=\left\lfloor\delta\left(\omega^{\prime}-\hat{H}_{0}\right) \hat{L}_{x} \delta\left(\omega-\hat{H}_{0}\right)\right\rfloor_{W} .
$$

Introducing into Eq. (4.17) the Fourier representations of the two $\delta$ functions and transforming to center-of-mass and relative coordinates one obtains

$$
\begin{aligned}
{\left[L_{x}\left(\omega, \omega^{\prime}\right)\right]_{W}=} & \iint \frac{d T d \tau}{(2 \pi \hbar)^{2}} e^{2 i E T} e^{i \varepsilon^{\tau}{ }_{2}} \\
& \times\left[e^{-i H_{0} T} L_{x}\left(\frac{\tau}{2}\right) e^{-i H_{0} T}\right]_{W},
\end{aligned}
$$

with

$$
E=\frac{\omega+\omega^{\prime}}{2}, \quad \varepsilon=\omega-\omega^{\prime}
$$

and

$$
O(t)=e^{i H_{0} t} O(0) e^{-i H_{0} t} .
$$

To lowest order in $\hbar$ we replace the triple operator product in Eq. (4.18) by the product of their Wigner transforms [6],

$$
\lim _{\hbar \rightarrow 0}\left[e^{-i H_{0} T} L_{x}\left(\frac{\tau}{2}\right) e^{-i H_{0} T}\right]_{W}=e^{-i 2 H_{0 \mathrm{cl}} T} L_{x}^{\mathrm{cl}}\left(\frac{\tau}{2}\right),
$$

and therefore

$$
\begin{aligned}
{\left[L_{x}\left(\omega, \omega^{\prime}\right)\right]_{W} } & =L_{x}(E, \varepsilon, \mathbf{R}, \mathbf{p}) \\
& =\frac{1}{2} \delta\left(E-H_{0 \mathrm{cl}}\right) \int \frac{d \tau}{(2 \pi \hbar)} e^{i \varepsilon \tau / 2 \hbar} L_{x}^{\mathrm{cl}}\left(\frac{\tau}{2}\right),
\end{aligned}
$$

with

$$
L_{x}^{\mathrm{cl}}(t)=R_{y}(t) p_{z}(t)-R_{z}(t) p_{y}(t)
$$

At this point the choice of our approximate self-consistent potential of harmonic-oscillator form [see Eq. (2.19)] turns out to be very helpful, since the classical trajectories in Eq. (4.22) can be given analytically:

$$
\begin{gathered}
R_{i}(t)=R_{i} \cos \left(\hbar \omega_{i} t\right)+\frac{p_{i}}{m \omega_{i}} \cos \left(\hbar \omega_{i} t\right), \\
p_{i}(t)=p_{i} \cos \left(\hbar \omega_{i} t\right)+m \omega_{i} R_{i} \cos \left(\hbar \omega_{i} t\right),
\end{gathered}
$$

with $i=x, y, z$. 
In the phase-space integral of Eq. (4.15), for reasons of symmetry, only the diagonal terms of $L_{x}^{\mathrm{cl}} L_{x}^{\mathrm{cl}}(t)$ survive and therefore we obtain

$$
\begin{aligned}
& \int \frac{d^{3} R d^{3} p}{(2 \pi \hbar)^{3}} L_{x}^{\mathrm{cl}} L_{x}^{\mathrm{cl}}(t) \\
& =\int d^{3} R \rho^{\mathrm{TF}}(\mathbf{R})\left(R_{y}^{2}+R_{z}^{2}\right) \cos \left(\hbar \omega_{y} t\right) \cos \left(\hbar \omega_{z} t\right) \\
& +\left(R_{y}^{2} \frac{\omega_{y}}{\omega_{z}}+R_{z}^{2} \frac{\omega_{z}}{\omega_{y}}\right) \sin \left(\hbar \omega_{y} t\right) \sin \left(\hbar \omega_{z} t\right)
\end{aligned}
$$

where

$$
\rho^{\mathrm{TF}}=\frac{1}{6 \pi^{2}}\left(\frac{2 m}{\hbar^{2}}(E-V)\right)^{3 / 2}
$$

is the density in the TF approximation [see Eq. (2.5)].

The product of cosine and sine in Eq. (4.25) can be expressed in terms of the cosine of the sum and difference of the arguments and then the $\tau$ integral in Eq. (4.23) can be performed. This leads to $\delta$ functions, which allows one to perform the $\varepsilon$ integral also. Furthermore, as shown by Migdal [7],

$$
F_{+}(E, \varepsilon) \approx\left[1-G\left(\frac{\varepsilon}{2 \Delta}\right)\right] \delta(E-\mu),
$$

where [see Eq. (3.5)]

$$
\Delta=\Delta\left(\varepsilon_{F}\right)
$$

and

$$
G(x)=\frac{\operatorname{arcsinh}(x)}{x \sqrt{1+x^{2}}} .
$$

Finally one obtains for the IB part of the moment of inertia the following analytical expression $[7,8]$ :

$$
\Theta_{\mathrm{IB}}=\Theta_{\text {rigid }}\left(1-\frac{G_{+} \omega_{-}^{2}+G_{-} \omega_{+}^{2}}{\omega_{-}^{2}+\omega_{+}^{2}}\right),
$$

where

$$
\omega_{ \pm}=\omega_{y} \pm \omega_{z}, \quad G_{ \pm}=G\left(\frac{\hbar \omega_{ \pm}}{2 \Delta}\right),
$$

and

$$
\Theta_{\text {rigid }}=\left(\frac{\mu^{4}}{24 \hbar^{3}}\right) \frac{\left(\omega_{y}^{2}+\omega_{z}^{2}\right)}{\omega_{z}^{3} \omega_{x} \omega_{y}^{3}}
$$

is the moment of inertia of rigid rotation. From Eq. (4.29) we see that

$$
\lim _{\Delta \rightarrow 0} \Theta_{\mathrm{IB}}=\Theta_{\text {rigid }}, \quad \lim _{\Delta \rightarrow \infty} \Theta_{\mathrm{IB}}=0
$$

The latter result is clearly unphysical and we will see how taking account of the reaction of the pair field to the rotation will reestablish the physical situation.

\section{B. The Migdal term}

The density response corresponding to the Migdal term is obtained from Eq. (4.6):

$$
\left(\rho_{1 M}\right)_{n, n^{\prime}}=\frac{\xi_{n} \Delta_{1 n n^{\prime}} \Delta_{0 n^{\prime}}+\Delta_{0 n} \Delta_{1 n n^{\prime}}^{*} \xi_{n^{\prime}}}{2 E_{n} E_{n^{\prime}}\left(E_{n}+E_{n^{\prime}}\right)} .
$$

In Eq. (4.32) we need to know $\Delta_{1}$ which we can gain from Eq. (4.7) in the following way. In the limit $\tau^{\prime} \rightarrow \tau^{+}$we obtain from $F_{1}^{+}$the anomalous density $\kappa_{1}^{+}$,

$$
\left(\kappa_{1}^{+}\right)_{n n^{\prime}}=-\frac{\xi_{n} H_{1 n n^{\prime}}^{*} \Delta_{0 n^{\prime}}+\Delta_{0 n} H_{1 n n^{\prime}} \xi_{n^{\prime}}+\Delta_{0 n} \Delta_{1 n n^{\prime}} \Delta_{0 n^{\prime}}-\left(E_{n} E_{n^{\prime}}+\xi_{n} \xi_{n^{\prime}}\right) \Delta_{1 n n^{\prime}}^{*}}{2 E_{n} E_{n^{\prime}}\left(E_{n}+E_{n^{\prime}}\right)} .
$$

In analogy with the nonrotating case where $\kappa_{0}=\Delta / 2 E$, we also have

$$
\left(\kappa_{1}^{+}\right)_{n n^{\prime}}=-\Delta_{1 n n^{\prime}}^{*}\left(\frac{1}{4 E_{n}}+\frac{1}{4 E_{n^{\prime}}}\right) .
$$

This relation stems from the fact that the quasiparticle energies contain the gap only in the form $\Delta \Delta^{*}$ and therefore there is no further first-order correction, since in our case the external field is a time-odd operator and thus

$$
\Delta_{1}^{*}=-\Delta_{1} \equiv-i \Omega \chi
$$

Equating (4.33) and (4.34) yields

$$
\frac{2 \xi_{n} H_{1 n n^{\prime}}^{*} \Delta_{0 n^{\prime}}+2 \Delta_{0 n} H_{1 n n^{\prime}} \xi_{n^{\prime}}+2 \Delta_{0 n} \Delta_{1 n n^{\prime}} \Delta_{0 n^{\prime}}+\left[\Delta_{0 n}^{2}+\Delta_{0 n^{\prime}}^{2}+\left(\xi_{n}-\xi_{n^{\prime}}\right)^{2}\right] \Delta_{1 n n^{\prime}}^{*}}{2 E_{n} E_{n^{\prime}}\left(E_{n}+E_{n^{\prime}}\right)}=0
$$


At this point we again exploit the fact that expression (4.36) is strongly peaked around the Fermi energy surface. Following [7], in analogy with Eq. (4.27), we have

$$
\left[E_{n} E_{n^{\prime}}\left(E_{n}+E_{n^{\prime}}\right)\right]^{-1} \approx \frac{1}{\Delta^{2}} G\left(\frac{\varepsilon_{n}-\varepsilon_{n^{\prime}}}{2 \Delta}\right) \delta\left(\frac{\varepsilon_{n}+\varepsilon_{n^{\prime}}}{2}-\mu\right) .
$$

With Eq. (4.35) we then obtain for Eq. (4.36)

$$
\begin{aligned}
& {\left[\frac{\left\langle n\left|\dot{L}_{x}\right| n^{\prime}\right\rangle}{2 \Delta}+\left(\frac{\varepsilon_{n}-\varepsilon_{n^{\prime}}}{2 \Delta}\right)^{2} \chi_{n n^{\prime}}\right] G\left(\frac{\varepsilon_{n}-\varepsilon_{n^{\prime}}}{2 \Delta}\right) \delta\left(\frac{\varepsilon_{n}+\varepsilon_{n^{\prime}}}{2}-\mu\right)} \\
& \quad=0
\end{aligned}
$$

where $\dot{L}_{x}$ stands for the time derivative of $L_{x}$. Summing on $n$ and $n^{\prime}$ and following exactly the same line of semiclassical approximations as the ones used for the derivation of $\Theta_{\mathrm{IB}}$, one arrives at the following relation [8]:

$$
\int_{-\infty}^{+\infty} d \tau G(\tau) \int \frac{d^{3} p}{(2 \pi \hbar)^{3}}\left[\frac{\dot{L}_{x}^{\mathrm{cl}}}{2 \Delta}-\frac{\ddot{\chi}(\tau)}{4 \Delta^{2}}\right] \delta\left(\mu-H_{0 \mathrm{cl}}\right)=0
$$

where $G(\tau)$ is the Fourier transform of $G(x)$ [Eq. (4.28)].

For the potential in Eq. (2.19), Eq. (4.39) is solved by

$$
\chi(\mathbf{R})=\alpha R_{y} R_{z},
$$

with

$$
\alpha=-2 \Delta m \omega_{+} \omega_{-} \frac{G_{+}+G_{-}}{\omega_{+}^{2} G_{+}+\omega_{-}^{2} G_{-}} .
$$

Inserting this solution into Eq. (4.32) leads for the Migdal part of the moment of inertia to $[7,8]$

$$
\Theta_{M}=\Theta_{\text {rigid }} \frac{\omega_{+}^{2} \omega_{+}^{2}}{\omega_{+}^{2}+\omega_{-}^{2}} \frac{\left(G_{+}+G_{-}\right)^{2}}{\omega_{+}^{2} G_{+}+\omega_{-}^{2} G_{-}} .
$$

Together with Eq. (4.29) the expression for the moment of inertia is now complete. Let us again mention that we neglected the temperature dependence except that contained in $\Delta=\Delta(T)$, since all other $T$ dependence for $T<T_{c}$ is negligible. The moment of inertia can then be calculated as a function of deformation and temperature. For example, it is immediately obvious that for $\Delta \rightarrow \infty$ Eq. (4.42) yields the irrotational-flow value,

$$
\lim _{\Delta \rightarrow \infty} \Theta_{M}=\Theta_{\text {irrot }}=\Theta_{\text {rigid }}\left(\frac{\omega_{y}^{2}-\omega_{z}^{2}}{\omega_{y}^{2}+\omega_{z}^{2}}\right)^{2},
$$

and therefore

$$
\lim _{\Delta \rightarrow \infty} \Theta=\lim _{\Delta \rightarrow \infty}\left(\Theta_{\mathrm{I}-\mathrm{B}}+\Theta_{M}\right)=\Theta_{\text {irrot }},
$$

which is the correct physical result.

\section{CURRENT DISTRIBUTION}

Other quantities that may also be interesting from the experimental point of view are the current distributions of the superfluid rotating gas. Indeed, after a sudden switching off of the (rotating) trap, the atoms will expand, keeping memory of their rotational state. So if the velocity distribution of the expanding atoms can be measured, one may be able to deduce the rotational motion the atoms had before the trap was taken away. The current distribution, as we will see, like the moment of inertia, depends strongly on the superfluid state of the gas. In order to calculate the current distribution we first write down the Wigner function of the density response which can easily be read off from the formulas given in Sec. IV. In obvious notation we obtain [8]

$$
\begin{aligned}
\rho_{1 \mathrm{IB}}(\mathbf{R}, \mathbf{p})= & \Omega[\mathbf{R} \times \mathbf{p}]_{x} \delta\left(\mu-H_{0 \mathrm{cl}}\right) \\
& -\Omega\left[\frac{\omega_{+} G_{-}-\omega_{-} G_{+}}{2 \omega_{z}} R_{y} p_{z}\right. \\
& \left.-\frac{\omega_{+} G_{-}+\omega_{-} G_{+}}{2 \omega_{y}} R_{z} p_{y}\right] \delta\left(\mu-H_{0 \mathrm{cl}}\right),
\end{aligned}
$$

$$
\begin{aligned}
\rho_{1 M}(\mathbf{R}, \mathbf{p})= & \frac{\hbar^{2}}{2 m} \frac{\alpha}{\Delta} \Omega\left[\frac{\omega_{+} G_{+}-\omega_{-} G_{-}}{\omega_{z}} R_{y} p_{z}\right. \\
& \left.+\frac{\omega_{+} G_{+}+\omega_{-} G_{-}}{\omega_{y}} R_{z} p_{y}\right] \delta\left(\mu-H_{0 \mathrm{cl}}\right) .
\end{aligned}
$$

With the usual definition of the current

$$
\mathbf{j}(\mathbf{R})=\int \frac{d^{3} p}{(2 \pi \hbar)^{3}} \frac{\mathbf{p}}{m} \rho(\mathbf{R}, \mathbf{p}),
$$

one obtains

$$
\begin{array}{r}
j_{y}^{\mathrm{IB}}=-\rho_{\mathrm{TF}}(\mathbf{R}) R_{z} \Omega\left[1-\frac{\omega_{+} G_{-}+\omega_{-} G_{+}}{2 \omega_{y}}\right], \\
j_{z}^{\mathrm{IB}}=-\rho_{\mathrm{TF}}(\mathbf{R}) R_{y} \Omega\left[1-\frac{\omega_{+} G_{-}-\omega_{-} G_{+}}{2 \omega_{z}}\right], \\
j_{y}^{M}=-\rho_{\mathrm{TF}}(\mathbf{R}) R_{z} \Omega\left[\frac{\omega_{+} G_{-}\left(G_{-}+G_{+}\right)}{\omega_{+}^{2} G_{+}+\omega_{-}^{2} G_{-}} \frac{\omega_{+} G_{-}+\omega_{-} G_{+}}{\omega_{y}}\right], \\
j_{z}^{M}=-\rho_{\mathrm{TF}}(\mathbf{R}) R_{y} \Omega\left[\frac{\omega_{+} \omega_{-}\left(G_{-}+G_{+}\right)}{\omega_{+}^{2} G_{+}+\omega_{-}^{2} G_{-}} \frac{\omega_{+} G_{-}-\omega_{-} G_{+}}{\omega_{z}}\right],
\end{array}
$$

with, of course, $j_{x}=0$. Again we see that in the limit $\Delta$ $\rightarrow \infty$ the current approaches the correct irrotational-flow limit,

$$
\mathbf{j} \underset{\Delta \rightarrow \infty}{\longrightarrow}-2 \rho_{\mathrm{TF}} \Omega \frac{\omega_{y}^{2}-\omega_{z}^{2}}{\omega_{y}^{2}+\omega_{z}^{2}} \nabla\left(r_{y} r_{z}\right),
$$



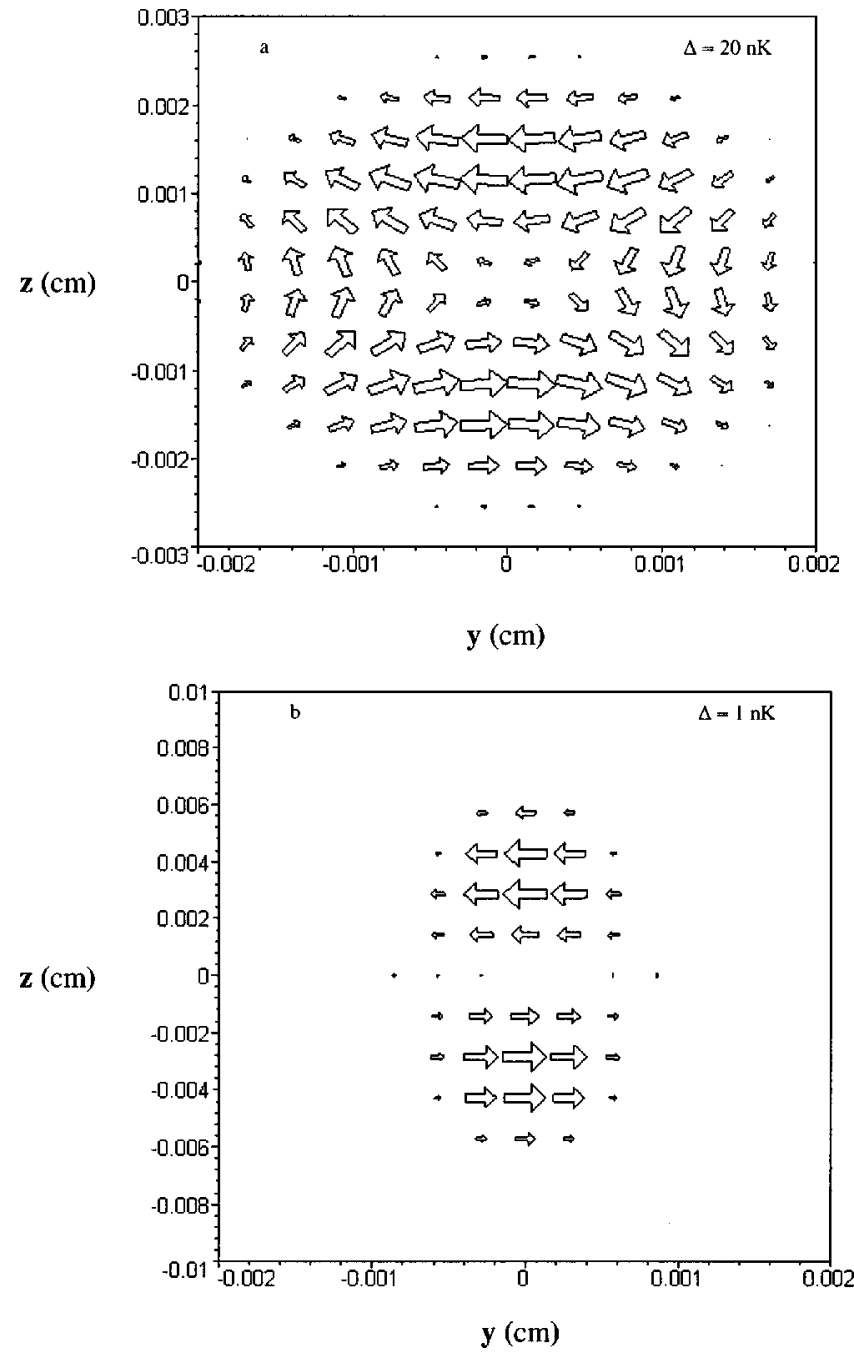

FIG. 4. The current distribution for the two extreme cases of irrotational (a) and rigid-body (b) flow in the laboratory frame. In both cases the deformation parameters are set to $\sigma=1 / 8, \delta=0.8$ and the angular frequency $\Omega$ around the $x$ axis to $1 \mathrm{nK}$.

whereas in the limit of $\Delta \rightarrow 0$ we obtain a rigid-body current. As we have seen for $\Theta$, as a function of temperature and deformation, we can easily go from one limit to the other.

\section{RESULTS AND CONCLUSION}

We show in Figs. 4(a) and 4(b) the current distribution for the two extreme cases of irrotational and rigid-body flow in the laboratory frame. We see that the flow pattern is completely different in the two cases. In Fig. 4(b) the flow pattern clearly corresponds to rigid rotation of an ellipsoid with the long axis in the $z$ direction. Figure 4(a) represents a typical irrotational-flow pattern well known from hydrodynamics. As a function of temperature one can pass continuously from one flow pattern to the other. The point we want to make is that for small deformations $\delta$, as can be seen from Eq. (5.6) there is almost no irrotational current for low temperatures and this will be reflected in a very low rotational energy, as we will discuss now.

In Fig. 5 we show $\Theta$ as a function of $\Delta(T)$ and with Fig.

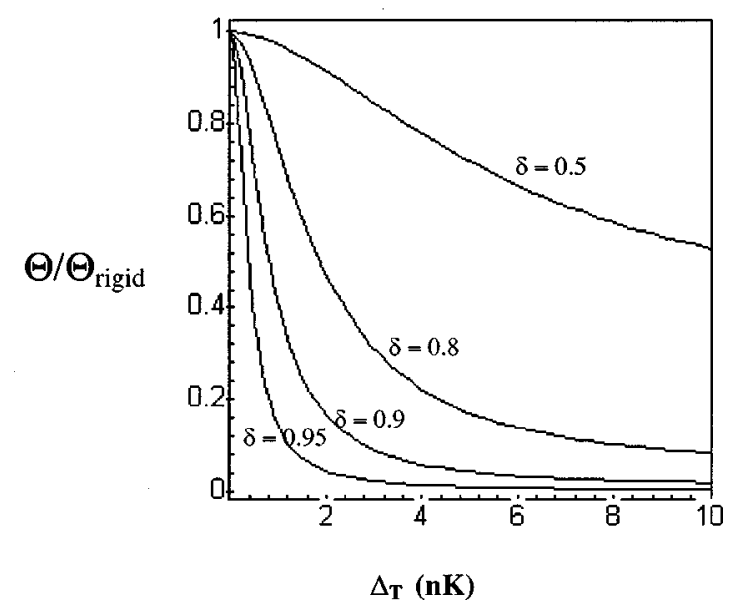

FIG. 5. The moment of inertia as a function of the gap for different values of the deformation $\delta=\omega_{z} / \omega_{y}$ and $\sigma=1 / 8$.

3 also as a function of $T$. We see that for a typical eccentricity $\delta=\omega_{z} / \omega_{y}=0.8$ the moment of inertia changes, as a function of temperature, by large factors. At $T \approx 0$ the gap values found in this paper are in the range of $10-20 \mathrm{nK}$ and therefore the moment of inertia is close to its irrotational-flow limit. This actually means that the moment of inertia is very small with respect to its rigid-body value, since for $\delta \rightarrow 1$, i.e., for spherical symmetry around the rotational axis $(x$ axis), the moment of inertia goes to zero [see Eqs. (4.43) and (4.44)]. Consequently in this case the gas is not following the rotation of the trap at all. However, increasing the temperature, i.e., decreasing the gap value, has a dramatic influence on the rotational motion of the gas, since in the range $0<T$ $<T_{c}$ the moment of inertia rises very steeply, attaining its rigid-body value for $T=T_{c}$. In this limit the gas rotates as a whole with the same angular frequency as the trap. The abruptness of the rise is the more pronounced the smaller the eccentricity $\delta$ (see Fig. 5). Experimentally, nondestructive or expansion imaging can be used to watch the gas rotate and then the rotational energy

$$
E_{\mathrm{rot}}=\frac{\Theta}{2} \Omega^{2}
$$

can be obtained by integrating the angular velocity over the density profile. The rotational energy therefore directly follows the variation of the moment of inertia. One deduces that measurement of the variation as a function of $T$ of the rotational energy should be well within experimental possibilities, once the technique of putting the trap into rotation has been perfected.

In our discussion we have ignored the possibilities of vortex formation. The determination of the onset of instabilities versus vortex formation in a finite Fermi system is not a completely easy task and we will postpone such an investigation to future work. However, since the rotational frequencies $\Omega$ considered in this paper are much smaller than the oscillator constant $\omega_{0}\left(\Omega / \omega_{0} \ll 1\right)$, we think that our result will not be changed by the appearance of vortices. An indication can also come from the case of trapped bosons where 
vortices, depending somewhat on the number of atoms, do not appear for values $\Omega / \omega_{0}<0.5$ (see Ref. [2])

From the above discussion we see that it may well be within experimental reach to reveal an eventual superfluid state of the gas once the technique of putting the trap into rotation has been perfected. A closely related phenomenon to rotation is the so-called scissors mode which was originally discussed and found in deformed nuclei [17] and then proposed [18] and also very recently found [19] for trapped boson condensates. Suppose the trapped atomic system is rotating very slowly and suddenly the rotation of the deformed trap potential is stopped. Due to inertia the atomic cloud will continue rotating back and forth around the fixed trap position if the initial rotation was gentle enough. If for the purpose of a rough argument we suppose that this oscillatory motion has so small an amplitude that in a first approximation we can neglect shape distortions of the cloud, then, if the oscillations are in the harmonic regime, the frequency of the scissors mode is given by

$$
\omega_{S}=\sqrt{C / \Theta},
$$

where $C$ is the constant of the restoring force. The frequency $\omega_{S}$ will strongly depend on whether the system is in the superfluid state or not. In this way the above cited experiment has indeed unambiguously revealed that the Bose condensate is in a superfluid state [19]. It is evident that scissors modes could also be excited in trapped Fermi systems, as was already mentioned in [18]. Since in Fermi systems for temperatures $T \sim T_{c}$ one can suppose that the temperature dependence of the force constant is weak with respect to that of the moment of inertia $\Theta$, one will find a large difference between the values of $\omega_{S}$ in the superfluid and unpaired regimes (see Fig. 5). A more detailed investigation of the scissors mode for trapped fermions may be given in future work.

In summary we proposed in this work to measure the dynamics of a rotating trapped gas of atomic fermions as a function of temperature and deformation to detect whether the system is in a superfluid state or not. Quite detailed and quantitative calculations for the moment of inertia and velocity distributions have been presented. Other quantities well studied in the case of rotating superfluid nuclei [6] such as Yrast lines, even-odd effects, particle alignment, etc., may also become of interest in this case.

\section{ACKNOWLEDGMENTS}

We gratefully acknowledge very useful discussions with C. Gignoux, D. Guéry-Odelin, S. Stringari, and W. Zwerger and a careful reading of the manuscript by $\mathrm{M}$. Durand. One of us (X.V.) also acknowledges financial support from DGCYT (Spain) under Grant No. PB98-1247 and from the DGR (Catalonia) under Grant No. GR94-1022.

\section{APPENDIX}

In this Appendix we want to give a more refined semiclassical solution of the gap equation. Let us write the quantal version of Eq. (3.3) at $T=0$ in the BCS approximation [6],

$$
\Delta_{n}=\sum_{n^{\prime}}\left\langle n \bar{n}|v| n^{\prime} \bar{n}^{\prime}\right\rangle \Delta_{n^{\prime}}\left(\frac{\tanh \left(E_{n^{\prime}} / 2 T\right)}{2 E_{n^{\prime}}}-\frac{P}{2\left(\varepsilon_{n^{\prime}}-\varepsilon_{F}\right)}\right),
$$

where $n$ labels the states of the (spherical) harmonic oscillator with single-particle energies $\varepsilon_{n}$ and $\bar{n}$ is the time-reversed state. As usual, $E_{n}=\sqrt{\left(\varepsilon_{n}-\varepsilon_{F}\right)^{2}+\Delta_{n}^{2}}$ is the quasiparticle energy and $\left\langle n \bar{n}|v| n^{\prime} \bar{n}^{\prime}\right\rangle$ is the matrix element of the zerorange two-body force

$$
g \delta\left(\mathbf{r}-\mathbf{r}^{\prime}\right)
$$

Since what matters is the gap at the Fermi level and since for particle numbers of the order $10^{5}$ the degeneracy of the oscillator shells is very high, it seems a very reasonable approximation to replace all quantities in Eq. (A1) by their corresponding values averaged over the energy shell [Eqs. (3.5) and (3.6)]. Equation (A1) can then be written as

$$
\begin{aligned}
\Delta_{n}= & \int_{0}^{\infty} d \varepsilon^{\prime} g\left(\varepsilon^{\prime}\right) v\left(\varepsilon, \varepsilon^{\prime}\right) \Delta\left(\varepsilon^{\prime}\right)\left(\frac{\tanh \left[E\left(\epsilon^{\prime}\right) / 2 T\right]}{2 E\left(\varepsilon^{\prime}\right)}\right. \\
& \left.-\frac{P}{2\left(\varepsilon^{\prime}-\varepsilon_{F}\right)}\right)
\end{aligned}
$$

where $g(\varepsilon)$ is the level density (3.7) and $v\left(\varepsilon, \varepsilon^{\prime}\right)$ is the averaged two-body matrix element

$$
\begin{aligned}
v\left(\varepsilon, \varepsilon^{\prime}\right)= & \frac{1}{g(\varepsilon) g\left(\varepsilon^{\prime}\right)} \sum_{n, n^{\prime}} \delta\left(\varepsilon-\varepsilon_{n}\right) \delta\left(\varepsilon^{\prime}-\varepsilon_{n^{\prime}}\right) \\
& \times\left\langle n \bar{n}|v| n^{\prime} \bar{n}^{\prime}\right\rangle .
\end{aligned}
$$

At this stage one could try to solve the gap equation numerically. However, in view of the huge number of particles it is certainly a good approximation again to pass to the ThomasFermi limit. For the level density $g(\varepsilon)$ this is straightforwardly performed. The TF limit of Eq. (A4) can be obtained by locally summing over plane waves and we obtain

$$
\begin{aligned}
v\left(\varepsilon, \varepsilon^{\prime}\right)= & \frac{g}{g^{\mathrm{TF}}(\varepsilon) g^{\mathrm{TF}}\left(\varepsilon^{\prime}\right)}\left(\frac{2 m}{\hbar^{2}}\right)^{3} \frac{1}{4 \pi^{3}} \\
& \times \int_{0}^{\inf \left\langle r_{\varepsilon}, r_{\varepsilon^{\prime}}\right\rangle} d r r^{2} \sqrt{\varepsilon-V(r)} \sqrt{\varepsilon^{\prime}-V(r)},
\end{aligned}
$$

where $r_{\varepsilon}$ is the classical turning point given by $\varepsilon=V\left(r_{\varepsilon}\right)$ and $V(r)=m \omega^{2} r^{2} / 2$ is the harmonic-oscillator potential. We have made a numerical check that Eq. (A5) is indeed a good approximation to the quantal counterpart for the case of large particle numbers [20]. We notice that Eq. (A5) needs the TF approximation only in the nonsuperfluid state where it is well justified (see Sec. II). Having an expression for average level density and matrix element at hand, we can proceed to solve Eq. (A3). We will do this again in the limit $\Delta\left(\varepsilon_{F}\right) / \varepsilon_{F} \ll 1$ and obtain (see [11]) at $T=0$ 


$$
\Delta\left(\varepsilon_{F}\right)=8 \varepsilon_{F} \exp \left(-\frac{15 \pi^{2}}{64 k_{F}|a|}+I\left(\varepsilon_{F}\right)\right)
$$

with

$$
I\left(\varepsilon_{F}\right)=2 \int_{0}^{1} d x \frac{x^{5} v^{2}\left(x^{2} \varepsilon_{F}, \varepsilon_{F}\right) / v^{2}\left(\varepsilon_{F}, \varepsilon_{F}\right)-1}{1-x^{2}} .
$$

The integral $I\left(\varepsilon_{F}\right)$ is evaluated numerically and we obtain

$$
\Delta\left(\varepsilon_{F}\right)=8 \varepsilon_{F} e^{-2.447} e^{-15 \pi^{2} / 64 k_{F}|a|} .
$$

With $\varepsilon_{F}=983.67 \mathrm{nK}$, which corresponds to our "selfconsistent' harmonic solution, and $k_{F}|a|=0.56$ one obtains $\Delta\left(\varepsilon_{F}\right)=11.29 \mathrm{nK}$. This value is about $30 \%$ smaller than the one extracted in Eq. (3.9), which, however, in view of the roughness of the TF approximation can be considered as a rather satisfying consistency of the results.
[1] A. S. Parkins and D. F. Walls, Phys. Rep. 303, 1 (1998).

[2] F. Dalfovo, S. Giorgini, L. P. Pitaevskii, and S. Stringari, Rev. Mod. Phys. (to be published).

[3] E. R. I. Abraham, W. I. McAlexander, C. A. Sackett, and R. G. Hulet, Phys. Rev. Lett. 74, 1315 (1995); F. S. Cataliotti, E. A. Cornell, C. Fort, M. Inguscio, F. Marin, M. Prevedelli, L. Ricci, and G. M. Tino, Phys. Rev. A 57, 1136 (1998).

[4] B. De Marco and D. S. Jin, Science 285, 1703 (1999).

[5] M. Houbiers, R. Ferweda, H. T. C. Stoof, W. I. McAlexander, C. A. Sackett, and R. G. Hulet, Phys. Rev. A 56, 4864 (1997).

[6] P. Ring and P. Schuck, The Nuclear Many Body Problem (Springer, Berlin, 1980).

[7] A. B. Migdal, Nucl. Phys. 13, 655 (1959).

[8] M. Durand, P. Schuck, and J. Kunz, Nucl. Phys. A 439, 263 (1985).

[9] R. Bengtsson and P. Schuck, Phys. Lett. 89B, 321 (1980).

[10] C. C. Bradley, C. A. Sackett, J. J. Tollet, and R. G. Hulet, Phys. Rev. Lett. 75, 1687 (1995); C. C. Bradley, C. A. Sackett, and R. G. Hulet, ibid. 78, 985 (1997).

[11] H. Kucharek, P. Ring, and P. Schuck, Z. Phys. A 334, 119 (1989).

[12] P. Schuck and K. Taruishi, Phys. Lett. B 385, 12 (1996).

[13] E. M. Lifshitz and L. P. Pitaevskii, Statistical Physics Part 2. Theory of the Condensed State (Pergamon, Oxford, 1980).

[14] D. J. Thouless and J. G. Valatin, Nucl. Phys. 31, 211 (1962).

[15] S. T. Belyaev, Nucl. Phys. 24, 322 (1961).

[16] A. L. Fetter and J. D. Walecka, Quantum Theory of ManyParticle Systems (McGraw-Hill, New York, 1971).

[17] N. Lo Indice and F. Palumbo, Phys. Rev. Lett. 41, 1532 (1978); E. Lippari and S. Stingari, Phys. Lett. 130B, 139 (1983); J. Enders, H. Kaiser, P. von Neumann-Cosel, C. Rangacharyulu, and A. Richter, Phys. Rev. C 59, 1851 (1999).

[18] D. Guéry-Odelin and S. Stingari, Phys. Rev. Lett. 83, 4452 (1999).

[19] O. M. Marengo et al., e-print cond-mat/9911195.

[20] P. Schuck and X. Viñas (unpublished). 